\title{
Consumption Intention of Online Game based on Motivation Theory Wan-Lin Lee ${ }^{1, a}$ and Delphine Ya-Chu Chan ${ }^{1, b,{ }^{*}}$ \\ ${ }^{1}$ School of Business, Beijing Institute of Technology, Zhuhai, Guangdong, China alifeisfaith@163.com, bw-chu119@qq.com
}

${ }^{*}$ Corresponding author

Keywords: Motivation theory, Online game, Consumption intention.

\begin{abstract}
In view of the consumption potential of young online game players in China, this study collected 385 valid samples through questionnaire survey to explore the influencing factors of their consumption intention. The results show that the player's achievement experience and immersion experience have a significant positive impact on the trust at the same time, and the trust and social experience have a significant positive effect on the game consumption intention. This study also confirms the importance of the motivation theory for the consumption intention of online games.
\end{abstract}

\section{基于游戏动机理论的网络游戏消费意愿}

\author{
李万林 ${ }^{1, a}$, 詹雅竹 $1, \mathrm{~b},{ }^{*}$ \\ 1 北京理工大学珠海学院商学院, 珠海, 广东, 中国 \\ alifeisfaith@163.com, bw-chu119@qq.com \\ 通讯作者
}

关键词: 游戏动机理论; 网络游戏; 消费意愿

中文摘要. 鉴于中国网游年轻玩家的消费潜力, 本研究通过问卷调查法搜集385份有效样本, 探讨其消费意愿的影响因素。结果表明, 游戏中的成就体验与沉浸体验会正向影响玩家信任, 而信任和社交体验对于游戏消费意愿也具有正向显著影响, 由此验证了游戏动机理论对网游 消费意愿的重要性。

\section{1. 引言}

《2017年1-6月中国游戏产业报告》显示, 中国游戏市场收入在2017年上半年达997.8亿元， 同比增长率为 $26.7 \%$, 增幅是自 2008 年以来的最高 ${ }^{[1]}$ 。但是, 以中国游戏玩家的规模而言, 网 络游戏用户规模达到 4.22 亿, 占整体网民的 $56.1 \%$, 同比增长 $3.6 \%$, 相较于 2016 年 $6.7 \%$ 的增幅 反而下滑 ${ }^{[2]}$ 。由此可知, 虽然游戏市场带来的收入仍在不断增加, 但是游戏用户数量的增长 却放缓了脚步。因此, 当游戏市场依靠的大基数人口带来的优势逐渐消失时, 如何最大限度 地激发每位游戏用户的消费意愿，以获得更大的利润，则是游戏供应商必须考虑的问题。

本文基于Yee (2007)提出的游戏动机理论, 将网游玩家的动机分为成就体验、社交体验和 沉浸体验 ${ }^{[3]}$, 通过探讨大学生的游戏动机、信任与其消费意愿, 帮助游戏供应商发掘出激发 年轻玩家们的消费潜力的方法。 


\section{2. 文献回顾}

\section{1 游戏动机体验}

游戏动机是指驱动玩家进行游戏的因素，包括：成就(achievement)、社交(social)和沉浸 (immersion)体验。成就体验是指游戏玩家会在游戏的引导或自我的意识下给自身设置一定的 目标, 当这些目标被完成时, 玩家会从中获得满足感与愉悦感。社交体验是指玩家在游戏中 通过长期的情感互动与团队协作, 对互动双方产生了信赖、善意、好感等感情的体验。沉浸 体验是指玩家在游戏过程中试图体验不同的人生, 以满足他们进行角色扮演的欲望 ${ }^{[3-4]}$ 。

\section{2 信任}

信任(trust) 是长时间发展出来的直接经验, 表示网游玩家之间经过时间积累而存在相互 依靠与信赖的感情关系 ${ }^{[5]}$ 。

\section{3 消费意愿}

消费意愿(consumption intention)是指消费者购买某种产品或者服务的可能性, 表示玩家在 游戏过程中为获得某产品或服务而愿意消费的可能性 ${ }^{[6]}$ 。

过去研究显示, 网络游戏动机对于玩家信任有显著影响 ${ }^{[7]}$, 而且信任程度也会在认知层 面上影响消费意愿 ${ }^{[8]}$ 。因此, 本研究提出相关假设如下:

H1：(a)成就体验、(b)沉浸体验、(c)社交体验对信任有正向影响

H2：(a)成就体验、(b)沉浸体验、(c)社交体验对消费意愿有正向影响

$\mathrm{H} 3$ ：信任对消费意愿有正向影响

\section{3. 研究方法}

本研究指的游戏包括PC端网络游戏与移动端网络游戏, 不包括买断制的单机游戏。研究 对象为中国的大学生。本研究采用问卷调查法, 问卷发放选择在游戏玩家聚集的大型社交平 台 (如: 百度贴吧、游戏官方论坛等), 以确保受访者都是游戏玩家, 减少无效问卷的比例。

所有变量测题皆采用李克特五点量表，成就体验、社交体验与沉浸体验的测题参考自Yee (2007), 分别有6题、5 题、3题; 信任的测题参考自Dwyer et al. (1987), 有4题; 消费意愿的测 题参考自Wang (2016), 有4题。30个前测样本的信度分析结果显示, 所有变量的Cronbach' s $\alpha$ 在0.874至 0.931 之间, 确认信度无疑后即发放正式问卷。回收的450份正式问卷中, 剔除非调 查对象等无效样本后, 有效样本为 385 份, 有效回收率达 $85.56 \%$ 。其中, 有 $64.42 \%$ 的受访者 为女性, $35.58 \%$ 为男性; 受访者的每周游戏天数有 $52.21 \%$ 是每天, 每日平均游戏时长有 $57.92 \%$ 是在1-3小时内; 玩游戏的场所主要集中于寝室 (77.40\%); 每月可支配收入多集中于 $801-1200$ 元 $(32.32 \%)$; 而近三个月的充值金额约为 100 元以下 $(50.12 \%)$ 。

\section{4. 分析结果}

\section{1 信效度分析}

从表1可知, 所有变量的Cronbach' $\mathrm{s} \alpha>0.7$, Item-total correlation $>0.5$, Factor loading $>$ 0.7、Composite reliability $(\mathrm{CR})>0.7, \mathrm{AVE}>0.5$, 显示各变量有高度的内部一致性与良好的收 玫效度。表2可见各变量AVE开方后均大于变量之间的相关系数, 说明各变量间具有良好的区 别效度。 
表1 信度与收敛效度

\begin{tabular}{|c|c|c|c|c|c|}
\hline \multirow{2}{*}{ Construct \& Item } & \multicolumn{2}{|c|}{ Internal reliability } & \multicolumn{3}{|c|}{ Convergent validity } \\
\hline & Cronbach's $\alpha$ & Item-total correlation & Factor loading & $\mathrm{CR}$ & AVE \\
\hline \multicolumn{6}{|l|}{ Achievement } \\
\hline ACH1. 挑战自我 & .928 & .785 & .785 & .944 & .739 \\
\hline ACH2. 自我肯定 & & .868 & .875 & & \\
\hline ACH3．自我满足 & & .899 & .901 & & \\
\hline ACH4. 实现自我价值 & & .900 & .895 & & \\
\hline ACH5. 充分展示自己 & & .836 & .829 & & \\
\hline ACH6. 获得竞争快感 & & .863 & .866 & & \\
\hline \multicolumn{6}{|l|}{ Social } \\
\hline SOC1. 能和其他玩家沟通 & .909 & .863 & .863 & .933 & .735 \\
\hline SOC2. 能帮其他玩家解决问题 & & .881 & .885 & & \\
\hline SOC3. 能向其他玩家提供信息 & & .869 & .872 & & \\
\hline SOC4. 能和其他玩家交流感情 & & .837 & .831 & & \\
\hline SOC5. 能得到其他玩家的响应 & & .836 & .834 & & \\
\hline \multicolumn{6}{|l|}{ Immersion } \\
\hline IMM1. 可以尽情幻想 & .864 & .851 & .850 & .917 & .786 \\
\hline IMM2. 可以体验别的人生 & & .911 & .915 & & \\
\hline IMM3. 可以追求向往的世界 & & .896 & .894 & & \\
\hline \multicolumn{6}{|l|}{ Trust } \\
\hline TRU1. 可以信赖 & .870 & .804 & .797 & .913 & .723 \\
\hline TRU2. 可以关注我们的利益 & & .852 & .840 & & \\
\hline TRU3. 可以提供所需的商品 & & .855 & .867 & & \\
\hline TRU4. 可以提供所需的服务 & & .887 & .895 & & \\
\hline \multicolumn{6}{|l|}{ Consumption Intention } \\
\hline CON1. 会定期浏览商品 & .872 & .813 & .802 & .913 & .725 \\
\hline CON2. 会进行玩家交易活动 & & .865 & .872 & & \\
\hline CON3. 会寻找商品相关信息 & & .898 & .904 & & \\
\hline CON4. 会想买游戏提供的商品 & & .827 & .825 & & \\
\hline
\end{tabular}

\begin{tabular}{|c|c|c|c|c|c|}
\hline \multicolumn{6}{|c|}{ 表2 区别效度 } \\
\hline Constructs & 1 & 2 & 3 & 4 & 5 \\
\hline 1. ACH & .859 & & & & \\
\hline 2. SOC & $.331^{* *}$ & .857 & & & \\
\hline 3. IMM & $.688^{* *}$ & $.281^{* *}$ & .887 & & \\
\hline 4. TRU & $.332^{* *}$ & $.200^{* *}$ & $.359^{* *}$ & .851 & \\
\hline 5. $\mathrm{CON}$ & $.337^{* *}$ & $.465^{* *}$ & $.324^{* *}$ & $413^{* *}$ & .852 \\
\hline
\end{tabular}

Note: The square roots of average variance extracted (AVEs) are shown in bold on the diagonal of the matrix; off diagonal elements are the correlations between constructs.

\section{2 回归分析}

由图1可知, 成就体验对信任 $(\beta=0.139, p<0.01)$ 与沉浸体验 $(\beta=0.239, p<0.001)$ 对信任具有 正向显著影响, 社交体验对信任 $(\beta=0.087, p>0.05)$ 无显著影响。因此, H1(a)(b)假设成立, H1(c) 假设不成立, 即成就体验与沉浸体验越好, 玩家的信任会越高。相反地, 成就体验 $(\beta=0.075$, $p>0.05)$ 与沉浸体验 $(\beta=0.065, p>0.05)$ 对消费意愿均无显著影响, 社交体验 $(\beta=0.364, p<0.001)$ 与信任 $(\beta=0.292, p<0.001)$ 对消费意愿皆有正向显著影响。因此, H2(a)(b)假设不成立, H2(c)、 $\mathrm{H} 3$ 假设成立, 表示玩家在游戏中的社交体验和信任程度越高, 其消费意愿也会越高。 


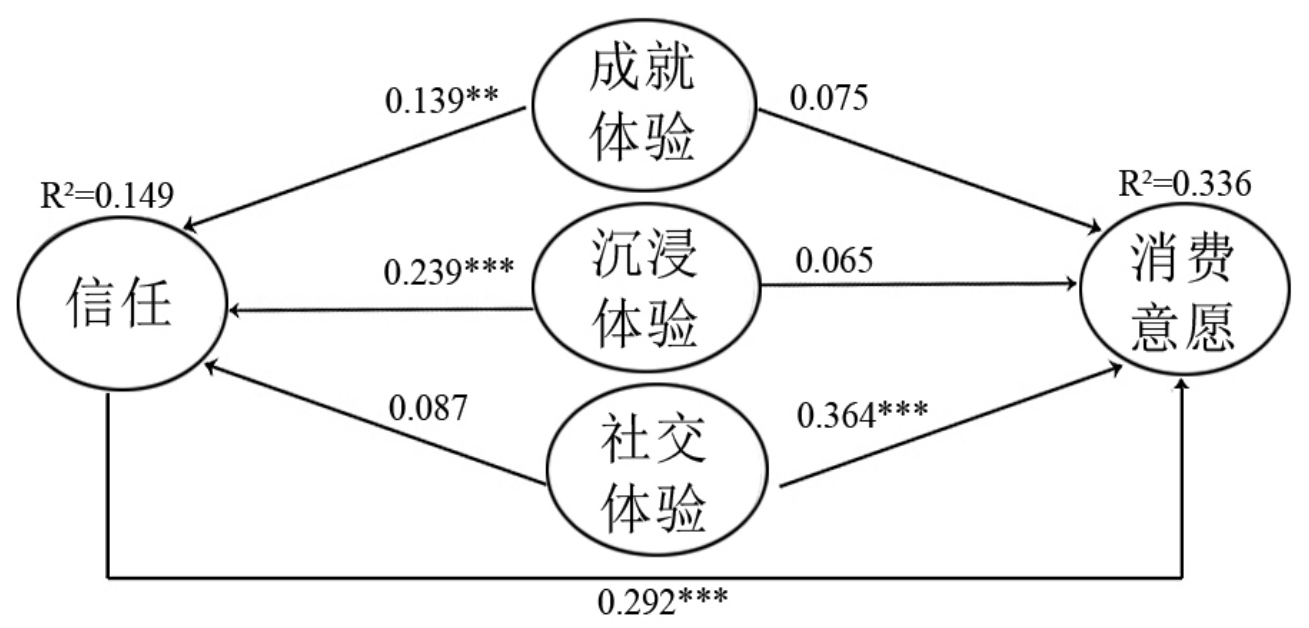

图 1 回归分析之路径系数图

\section{5. 结论}

游戏商投入到中国游戏产业的资源不断增加，许多游戏商都会将营销的重点放在游戏的 可玩性上。但是本研究结果表明, 在游戏动机理论中对消费意愿有显著影响的反而是社交体 验的好坏与否, 说明玩家对一个游戏的消费意愿更大程度取决于游戏中是否有足够良好的社 交环境。对游戏商而言，应该提供给玩家一个氛围良好的人际交往互动平台，让玩家在开放、 互助的积极游戏氛围中提升自己在游戏内的消费欲望。除此之外，也要保证游戏的可玩性以 提高玩家的成就体验与沉浸体验，从而进一步提升玩家对游戏的信任感。

\section{致谢}

本文为广东省高校创新人才类项目（人文社科） 《基于可持续发展的组织营销与消费研 究》(2015WQNCX191)的阶段性成果之一。

\section{References}

[1] GPC, CNG, and IDC, China gaming industry report, 2017.

[2] CNNIC, The $40^{\text {th }}$ China Statistical report on internet development, 2017.

[3] N. Yee, Motivations of play in online games, Cyberpsychology \& Behavior, vol. 9, pp. 772-775, 2007.

[4] R. Bartle, Heart, clubs, diamonds, spades: players who suit MUDs, Journal of Virtual Environments, vol. 2, pp. 235-242, 1996.

[5] F. R. Dwyer, P. H. Schurr, and S. Oh, Developing buyer-seller relationships, Journal of Marketing, vol. 51, pp. 11-27, 1987.

[6] Y. Wang, Research of the impact of players' interaction on the players' purchase intention in MMORPG, Nanjing: Nanjing University of Posts and Telecommunications, 2016.

[7] C. A. Gumussoy, Acceptance of the virtual item auctioning system in online games: the role of intrinsic motivation, extrinsic motivation, and trust, Human Factors \& Ergonomics in Manufacturing \& Service Industries, vol. 26, pp. 627-637, 2016.

[8] B. Lu, W. Fan, and M. Zhou, Social presence, trust, and social commerce purchase intention: an empirical research, Computers in Human Behavior, vol. 56, pp. 225-237, 2016. 